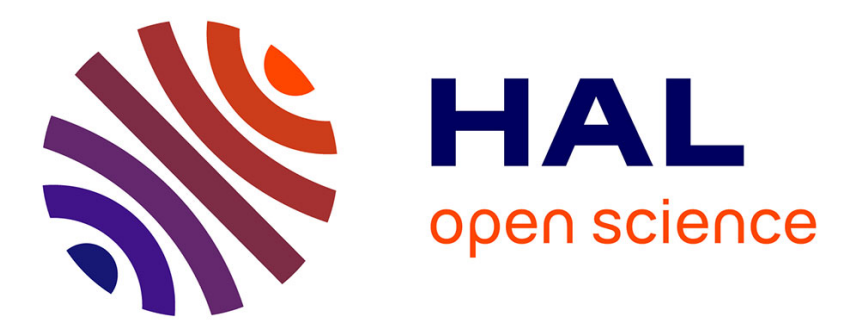

\title{
Possibilités d'une représentation conceptuelle de la situation normative.
}

Peter Stockinger

\section{To cite this version:}

Peter Stockinger. Possibilités d'une représentation conceptuelle de la situation normative. . Droit et Société, 1988, 8, pp.31-45. hal-00797745

\section{HAL Id: hal-00797745 \\ https://hal.science/hal-00797745}

Submitted on 7 Mar 2013

HAL is a multi-disciplinary open access archive for the deposit and dissemination of scientific research documents, whether they are published or not. The documents may come from teaching and research institutions in France or abroad, or from public or private research centers.
L'archive ouverte pluridisciplinaire HAL, est destinée au dépôt et à la diffusion de documents scientifiques de niveau recherche, publiés ou non, émanant des établissements d'enseignement et de recherche français ou étrangers, des laboratoires publics ou privés.

\section{(ㅇ)(1) $\$$}

Distributed under a Creative Commons Attribution - NonCommercial - NoDerivatives| 4.0 


\section{Possibilités d'une \\ représentation conceptuelle de la situation normative}

\section{Peter STOCKINGER *}

\section{Résumé}

Le but de cet essai consiste dans l'élaboration de quelques catégories conceptuelles typiques qui permettent la bonne compréhension d'une situation normative. Ainsi, on considérera, entre autres, des catégories telles que «autorité de norme», «sujet de norme», «norme», « régulation », etc. La notion «situation normative » est comprise dans un sens assez large incluant aussi ses usages possibles dans le langage naturel. Les sources théoriques de ce travail se trouvent d'une part dans I'article «The Foundation of Norms and Normative Statements » de G.H. von Wright et d'autre part dans le cadre conceptuel propre à la sémiotique structurale.

Summary

The aim of this essay is to elaborate some typical conceptual features that contribute to a good comprehension of a normative situation. Therefore, there are considered, amongst others, features like «norm authority », «norm subject», «norm», «regulation» and so on. The notion «normative situation » is understood in a quite broad sense including also corresponding expressions in natural language. The theoretical assumptions are basically founded on the one side on the article «The foundation of norms and normative statements » of G.H. von Wright and on the other side on the conceptual framework of structural semiotics.

\section{1}

Comme l'indique le titre de notre étude, nous essaierons ici de mettre en place différentes catégories conceptuelles (ou, comme on dit aussi, cognitives) permettant de saisir quelques aspects cen-

\section{(p. $31-45)^{\circ}$}


Peter Stockinger

Possibilité d'une représentation conceptuelle de la situation normative
1. G.H. von Wright, «The Foundation of Norms and Normative Statements », Philosophical Papers, Oxford, Blackwell, 1983, p. 67-82.

2. «By a normative statement I understand a statement to the effect that something ought to or may or must not be done. Such a statement is often, but not necessarily, elliptic or incomplete unless it says by whom this thing ought (may, must not) be done and whether it ought to (may, must not) be done generally or on some particular occasion, unconditionally or provided certain conditions are fulfilled », (op. cit., p. 67). traux (ou jugés tels) lors du processus de la mise en norme du comportement d'un sujet quelconque.

Ceci dit, nous ne considérerons pas une situation normative dans une optique restreinte à la science du droit. Ce qui nous intéresse plutôt, c'est la prise en charge d'une situation normative par le langage naturel, par le récit, le discours, la conversation, etc. Cependant, une telle position ne saurait faire abstraction des apports de la théorie des obligations en droit. Elle considère seulement I'obligation au sens juridique du terme comme un cas spécifique parmi la grande variété des situations normatives telle qu'elle est présente dans une société, une culture ou encore une époque donnée.

\section{II}

Comme point de départ de nos investigations dans le domaine des situations normatives, nous prenons l'article très riche "The Foundation of Norms and Normative Statements » de G.H. von Wright ${ }^{1}$.

Le «normative statement », qui a la forme d'une proposition au sens logique du terme, est défini comme suit :

«Par énoncé normatif, j'entends un énoncé dont il résulte que quelque chose doit, peut, ou ne doit pas être fait. Un tel énoncé est souvent, mais non nécessairement elliptique ou incomplet, à moins qu'il ne spécifie par qui ce quelque chose doit (peut, ne doit pas) être fait et si cela doit (peut, ne doit pas) être fait en général, ou seulement en certaines circonstances, conditionnellement ou inconditionnellement $\gg^{2}$.

Suivant cette définition, on peut déjà retenir quelques traits qui sont en fait des catégories conceptuelles permettant au moins une première compréhension globale de ce qu'est une situation normative :

1) un acte qui est le plus souvent de nature verbale mais qui peut être aussi non-verbale (voir, par exemple, le policier qui oblige avec un coup de sifflet ou un geste de son bras le conducteur d'une voiture à s'arrêter) ;

2 ) un objet de cet acte, qui est la norme ;

3) le domaine auquel la norme s'applique ;

4) la portée de la norme ;

5) I'applicabilité ou la condition de l'application de la norme ;

6) la source ou le destinateur de la proposition normative, soit, dans la terminologie de von Wright, l'autorité de norme ;

7) la cible ou le destinataire de la proposition normative, qui est le sujet de norme. 
À ces sept catégories il faut encore ajouter le lieu et le moment/la durée où naît, se déroule, se modifie et s'éteint une situation normative.

À part la mise en place des catégories conceptuelles permettant la compréhension des situations normatives, il se pose encore le problème de la fondation de l'acte normatif et de la norme ellemême, donc de leur «base de vérité » («truth-ground » selon von Wright ${ }^{3}$ ). Au lieu de se contenter d'attribuer aux actes normatifs d'une manière quasi mécanique les valeurs «vrai » et «faux» (pendant tel ou tel intervalle temporel ou spatial, pour tel ou tel fait, etc.), on peut aussi s'interroger sur «the general form and nature of the truth-grounds of such statements $\gg 4$.

Imaginons un policier qui arrête le conducteur d'une voiture en énonçant : «Vos papiers ! » II s'agit là évidemment d'une proposition «elliptique » puisqu'elle équivaut à l'acte normatif :

«Je vous commande de me montrer vos papiers, parce que... »

En pareil cas, tout ce qui suit le «parce que» sert à fonder l'acte normatif.

Un premier type de fondation - assez trivial et peu informatif repose sur une déduction dont la logique déontique fournit les lois inférentielles ${ }^{5}$. Le policier peut, en effet, affirmer son ordre : «Vos papiers », comme suit :

«... parce que vous êtes obligé de vous arrêter ; et puisque vous vous êtes arrêté, vous devez me montrer (aussi) vos papiers - donc : je vous commande de me montrer vos papiers. »

On a donc ici l'exemple d'une fondation s'appuyant sur une inférence déontique :

1) $O(p):$ : s'il y a obligation pour $x$ de faire $p$

2) $p(O(q)$ :] et si $x$ fait $p$, $x$ est aussi obligé de faire $q$

3) $O(q)$ :] alors $x$ est obligé de faire $q$.

Mais le policier peut aussi argumenter :

«... parce que j'ai le droit de vous commander de me montrer vos papiers. »

II s'agit ici, suivant von Wright ${ }^{6}$, d'une fondation normative d'une norme. La fondation normative présuppose des normes de compétence (au sens non-sémiotique) permettant de constater la validité ou la non-validité de tel ou tel acte normatif et donc de l'existence de telle ou telle norme: «A norm has a normative foundation if, and only if, there exists a competence norm which permits the act of issuing this norm to the authority, who actually has issued it $\gg 7$.

Depuis le travail d'E. Landowski, «Pour une approche sémiotique et narrative du droit ${ }^{8} 8$, le mécanisme de ce type de fondation

3. Ibid.

4. Ibid.

5. Voir, par exemple : R. Hilpinen (ed.), New Studies in Deontic Logic, Dordrecht, Reidel, 1981.

6. Op. cit., p. 70.

7. Ibid.

8. E. Landowski, « Pour une approche sémiotique et narrative du droit », Actes SémiotiquesDocuments, VIII, 71, 1986. 
Peter Stockinger Possibilité d'une représentation conceptuelle de la situation normative
9. G.H. von Wright, op. cit., p. 73. 10. Ibid. est bien connu en sémiotique structurale : il s'agit en effet d'une récursivité par emboîtement d'un rôle actantiel, celui du destinateur (ici, l'autorité de norme), dans un autre rôle actantiel, celui du destinateur-délégué (entretenant avec le premier rôle une identité fonctionnelle), et ainsi de suite.

Si le policier dit :

«... parce que je suis obligé moi-même de vous commander de me montrer vos papiers »,

il est clair qu'on est ici de nouveau devant une fondation normative, focalisée cette fois-ci sur l'obligation du policier et non pas sur son droit.

Un autre argument du policier peut être le suivant : règle. »

«... sinon je ne saurai pas si l'état de votre voiture est en

On a là une fondation anankastique, une fondation s'appuyant sur une condition nécessaire pour qu'un certain état de choses entre en existence : «In an anankastic statement there is mention of two states of affairs or events, of which the one is said to be (or not to be) a necessary condition of the other $\gg$.

Comme von Wright le remarque, les actes anankastiques doivent être distingués non seulement des actes normatifs proprement dits mais aussi de ce qu'il appelle les règles techniques. Une règle technique s'exprimerait comme suit dans l'argumentation de notre policier :

«Je vous commande de me montrer vos papiers, parce que je souhaite prendre connaissance de l'état de votre voiture, et c'est pour cela que j'ai besoin de vos papiers. »

Ici, I'ordre «vos papiers » renvoie au schème moyen/but: I'accomplissement d'un certain but se fait à l'aide de tel ou tel moyen. II s'agit ici donc d'une fondation par nécessité pratique.

Les différentes possibilités de fonder la norme par un acte se regroupent en fait autour de deux types principaux :

«... we could say that the idea of «ought » has two main sources. The one source is in the will of a commanding agent or norm-authority. The other is a double source in ends of human action and necessary connections between things $\gg 10$.

Un dernier point retenu par von Wright est finalement le fait que beaucoup de normes ne semblent être que des expressions, des mises en formes légales ou quasi-légales de principes moraux tels que «il faut tenir sa promesse», «il est interdit d'entretenir des relations sexuelles avec les membres de sa propre famille», «il faut respecter l'autre » ou encore «il ne faut pas mentir ». Ce genre de «lois» ne peuvent pas être considérées, en tant que telles, 
comme des normes, c'est-à-dire des obligations, interdictions ou permissions, bien qu'elles possèdent de toute évidence une «grammaire » ou une «forme logique » comparables avec celles-ci. Il s'agit ici, on le voit, d'une distinction similaire à celle entre les « règles » de la grammaire d'une langue et l'enseignement de ces règles qui les transforme en «normes » permettant de déterminer le «bon usage » de cette langue.

\section{III}

Comparons maintenant les différents types de fondation des normes avec les principes moraux. En fait, les principes moraux peuvent être considérés comme des sortes de méta-règles déterminant (globalement ou partiellement) l'équilibration des différentes formes de rapports intersubjectifs telles qu'elles sont définies (d'une manière explicite ou implicite) par des «contrats», des «conventions », des «jeux d'imitation » 11 ou encore, justement, des « règles normatives ».

II est curieux de voir converger sur ce point des recherches menées d'une part en anthropologie, sur l'échange, et d'autre part des recherches en pragmatique, sur la conversation ou l'interaction conversationnelle. La prohibition de l'inceste, par exemple, et les différents types de réglementation des échanges exogames ne peuvent pas seulement être interprétés comme des prescriptions, interdictions ou permissions au sens normatif du terme mais doivent être aussi considérés dans leur fonction régulatrice qui assure une cohérence nécessaire dans le fonctionnement des différentes pratiques d'une société ou d'une culture. Cette observation s'applique aussi au fameux «principe de coopérativité», introduit par H.P. Grize dans l'analyse des actes conversationnels. Comme J.-F. Bordron l'a bien remarqué :

«... un tel principe ne saurait être réduit à l'énoncé d'une norme sociale ou à un préjugé idéologique. C'est un principe d'intelligibilité qui (...) prend toute sa force si l'on accepte de le considérer comme un principe constitutif de la conversation, au sens précis où Kant dit, par exemple, que la catégorie de la causalité est constitutive des phénomènes $\gg 12$.

Dans leur ouvrage remarquable, Situations and Attitudes, J. Barwise et J. Perry ${ }^{13}$ introduisent la notion de la contrainte permettant de définir ce qui est le sens («meaning » en anglais) :

«A central claim (...) is that the meaning resides in the world in systematic relations of a special sort between different types of situations... These systematic constraints are what allow one situation to contain information about another. Attunement to these constraints is what allows an agent to pick up information from one situation about another $\gg 14$.
11. D. Lewis, Convention. A Philosophical Study, Cambridge (Mass), 1969.

12. J.-F. Bordron, « Remarque sur l'intelligibilité de l'échange », Actes SémiotiquesBulletin, VII, 30, 1984, p. 39-44. 13. J. Barwise et J. Perry, Situations and Attitudes, Cambridge (Mass), Bradford, 1983.

14. Op. cit., p. 61. 
Peter Stockinger Possibilité d'une représentation conceptuelle de la situation normative
Sans prendre en considération la métaphysique sous-jacente et propre à la sémantique formelle de J. Barwise et J. Perry - métaphysique qui reconnaît à un certain monde de référence (dit réalité) un statut privilégié et véridiquement déterminant -, on peut interpréter une «situation » ou un «type de situations » à la manière de G. Fauconnier comme un «espace mental » 15 propre à un locuteurnarrateur ou encore à un acteur (personnage) d'un récit. Un tel espace mental possède une certaine organisation conceptuelle ou sémantique interne, à l'aide de laquelle le locuteur ou le narrateur parvient à catégoriser et à narrativiser selon ses maximes, intentions ou buts les objets appartenant à un autre espace mental. Mais pour qu'il y ait organisation conceptuelle ou sémantique au sens fort du terme, il faut bien admettre des contraintes à la Barwise et Perry, c'est-à-dire des principes régulateurs déterminant la forme même d'une telle organisation.

Considérons dans cette perspective l'idée que les principes moraux sont des véritables mécanismes régulateurs. Si l'on envisage un acte épistémique en considérant le fait que l'obtention d'une information fiable présuppose tel ou tel critère de sincérité, d'informativité ou autre du même type, alors il est tout à fait raisonnable d'envisager que l'instanciation ou l'individuation du résultat d'un tel acte prenne la valeur «morale» du genre «tu ne dois pas mentir ». Une telle valeur «morale » peut être considérée ensuite comme le thème ou le propos de base d'une règle ou d'un type de règles à caractère obligatoire pour un individu ou un groupe d'individus. C'est seulement dans le dernier cas qu'on peut parler de normes ou de règles normatives. Si ces normes ou règles normatives sont institutionnalisées et prises en charge par une déontologie professionnelle particulière, alors on peut parler de normes au sens quasi-juridique ou juridique proprement dit; sinon, elles restent non-spécifiées et liées à un arrière-plan culturel, historique ou social général comme c'est le cas pour les règles de la politesse, de la bienséance, etc. L'hypothèse d'une fondation normative des normes est insuffisante pour expliquer ce double statut du contenu d'une norme: d'une part son statut régulateur pour telle ou telle conduite - statut qui dérive directement de la nécessité pratique d'admettre une certaine intelligibilité des rapports intersubjectifs ; d'autre part son statut proprement normatif liant, à I'aide de sanctions, une certaine conduite d'un agent quelconque en l'obligeant à admettre ou à respecter telle ou telle norme qui est conforme à un certain type de contraintes ou de régulations (logiques, nomiques, conventionnelles...).
15. G. Fauconnier, Espaces Mentaux, Paris, Minuit, 1984. 
1. Essayons maintenant de considérer plus en détail les différentes règles et catégories qui contribuent ensemble à la mise en scène d'une situation normative.

Nous avons dit, au début de la deuxième section, qu'une situation normative peut être exprimée par un certain type d'acte (de langage) dont la forme la plus typique est celle de l'ordre :

$$
\text { «Parle! » }
$$

Quelques remarques s'imposent ici :

a) l'ordre n'est qu'une forme particulière de ce que J. Searle appelle les actes ou énoncés directifs ${ }^{16}$. P. Larreya notamment a montré, dans son travail sur les auxiliaires modaux en anglais ${ }^{17}$, que toute une gamme d'actes particuliers entrent dans la catégorie des actes directifs. Ainsi nous avons, entre autres, l'acte d'avertissement :

«Parle, sinon on ne te comprendra pas!»

l'acte de suggestion :

(Je ne le sais pas :) « Parles-en avec Pierre ! »

l'acte d'instruction :

«Parle avec Pierre si tu veux obtenir son aide! »

«Mets la tarte au four pendant quarante minutes! »

P. Larreya distingue aussi d'autres actes plus spécifiques, mais les trois actes retenus nous suffisent pour saisir l'aspect conceptuel propre à un ordre. Ce que l'ordre manifeste, c'est le fait qu'un agent exprime son intention qu'un autre agent accomplisse une certaine action. Suivant von Wright, l'ordre a sa source dans la volonté de l'agent qui commande, c'est-à-dire dans la volonté de «l'autorité de norme». L'avertissement, la suggestion et l'instruction, par contre, se fondent sur une nécessité pratique visant la réalisation du but d'une certaine action, ou l'entrée en existence d'un certain état de choses. La suggestion peut être considérée, selon $P$. Larreya, comme une forme particulière du conseil en ce sens qu'elle propose une alternative parmi d'autres pour atteindre un certain but. L'instruction ressemble à une règle technique ou, de nouveau, à un conseil. Entendu dans ce dernier sens (instruction comme conseil), l'instruction présente un aspect directif «positif» ou «productif » par rapport à l'avertissement qui offre essentiellement un aspect «négatif » ou «préventif ». Nous ne nous proposons pas ici de dresser une typologie des différents actes directifs. Ce que nous voulons seulement retenir, c'est le fait que c'est le critère de la fondation qui confère aux actes directifs une significa-

16. Cf. P. Larreya, Le Possible et le Nécessaire, Paris, Nathan, 1984.

17. Op. cit., p. 226. 
Peter Stockinger Possibilité d'une représentation conceptuelle de la situation normative
18. Ibid.

19. J.-C. Anscombre, « Voulezvous dériver avec moi », Communications, 32, 1980, p. 61124. tion spécifique. II faut évidemment laisser ouverte la possibilité, à côté d'une fondation simple, de formes de fondation plus complexes liant à l'aide d'une relation de présupposition les deux types retenus par von Wright. Ainsi, dans une situation telle que celle-ci :

«Parle! Mais parle lentement, sinon on ne te comprendra pas »,

l'avertissement dérive d'un ordre, ce qui veut dire que la nécessité pratique déterminant la réussite d'une action est elle-même déterminée par la volonté de l'autorité de norme.

b) Un ordre et, a fortiori, un acte directif, peut s'exprimer linguistiquement par la voie de l'impératif, mais cela n'est pas nécessaire. L'impératif n'est qu'une possibilité grammaticale qu'offre une langue pour exprimer d'ailleurs non seulement un ordre, mais aussi une demande, une proposition, etc.

Le contenu propositionnel d'un ordre ou d'un acte directif en général peut en effet être pris en charge par toute une gamme d'expressions verbales, nominales, et d'autres encore du langage naturel. Il s'agit ici, selon P. Larreya, d'une «sémantisation totale ${ }^{18}$, par rapport à laquelle il faut encore distinguer, comme J.C. Anscombre l'a montré ${ }^{19}$, des actes dérivés marqués du type :

«Enfin, as-tu la gentillesse de parler ? »

et des actes dérivés allusifs du type :

«Évidemment, pas de réponse ».

Un acte normatif dérivé marqué ou allusif exprime encore l'intention d'un agent d'obliger un autre agent, mais cette intention n'est plus explicite - ce qui laisse au destinataire la liberté d'interpréter un tel acte aussi dans un sens non-normatif.

c) Un acte normatif comme l'ordre peut se manifester comme une forme énonciative, mais il peut être aussi le contenu ou le thème d'un énoncé ou d'un discours. II faut donc prendre en considération, indépendamment des stratégies énonciatives, le contenu propositionnel d'un énoncé ou d'une suite d'énoncés.

d) Une dernière remarque au sujet de l'acte d'ordre porte sur la «visée » de celui-ci. Pour le moment, nous ne retenons que le fait qu'un ordre peut avoir une visée «positive» («productive») ou «négative » («préventive») : dans le premier cas, on parlera d'une obligation; dans le second, d'une interdiction. Mises à part l'obligation et l'interdiction, il faut encore réserver une place à la permission, qui n'est pas un ordre (ou, du moins, pas un ordre ouvert et explicite) mais qui relève néanmoins de la compétence de l'autorité de norme puisque c'est elle qui détermine la liberté d'action du sujet de norme. Ceci dit, la permission fait elle aussi partie d'un acte normatif en tant que tel : le premier type n'englobe que les ordres «ouverts » ou «explicites » tels que l'obligation ou 
l'interdiction; le second inclut les ordres «couverts» ou «implicites », dont le prototype est justement la permission ; l'acte normatif en tant que tel représente enfin un type d'actes directifs plus général par rapport aux deux premiers dans la mesure où il les comprend comme des parties plus spécifiques en leur attribuant, comme nous le verrons, des positions précises.

Nous avons obtenu ainsi l'amorce d'une hiérarchie de types d'une certaine catégorie d'actes directifs - une hiérarchie qu'on pourrait encore étendre si l'on postule que les actes directifs représentent une sorte de "super-type » qui se scinde en des actes fondés sur la nécessité pratique (ce sont des actes directifs tels que le conseil, l'avertissement, l'instruction, etc.) ou sur la volonté de I'autorité de norme (ce sont des actes normatifs au sens strict). 2. A près avoir discuté différents problèmes relatifs à l'acte de langage qui exprime une situation normative, nous allons maintenant envisager les catégories permettant l'identification et la représentation d'une telle situation.

Les deux catégories qui sont centrales pour la description des situations normatives sont évidemment celles qui représentent d'une part les sujets impliqués dans de telles situations, d'autre part l'objet propre à l'interaction des deux catégories en question.

Avant de discuter ces deux catégories, notons qu'une approche structurale part de I'hypothèse fondamentale que les différents rôles et valeurs dont peuvent être dotés les sujets et les objets dans une situation déterminée sont distribués par un «centre organisateur » qui n'est autre que le prédicat, au sens abstrait du terme. Linguistiquement, un tel prédicat peut, entre autres, prendre la forme d'une expression verbale telle que «obliger quelqu'un », «contraindre quelqu'un», «astreindre quelqu'un », etc. Évidemment, le propos ou le thème de telles expressions peut être pris en charge non seulement par des phrases telles que:

$$
\begin{aligned}
& \text { "Jean oblige Paul à se rendre à Paris », } \\
& \text { «Jean contraint Paul à se rendre à Paris », }
\end{aligned}
$$

mais aussi par des unités transphrastiques, voir discursives. Ces dernières d'une part développent et complexifient davantage la structure interne de ce type de situations, et d'autre part les contextualisent, en ce sens qu'elles les mettent en rapport avec d'autres types de situations (narratives, volitionnelles, etc.) et les intègrent comme une partie spécifique dans une structure d'action ou d'événement plus globale.

Nous avons déjà introduit les deux termes «autorité de norme » et «sujet de norme » pour désigner l'actant qui «émet » une norme et l'actant qui la « reçoit ». Évidemment, l'introduction en quelque sorte ad hoc de deux rôles actantiels plus spécifiques est en soi peu satisfaisante parce qu'elle n'en fournit pas une définition explicite et rigoureuse. Ceci est d'ailleurs un problème que 
Peter Stockinger Possibilité d'une représentation conceptuelle de la situation normative rencontrent pratiquement toutes les théories actantielles, qu'elles soient linguistiques ou conceptuelles : souvent elles ne peuvent pas déterminer le contenu ou la nature exacte d'un rôle actantiel, ce qui s'exprime par des usages imprécis de cette catégorie dans les diverses applications. Bien qu'on puisse accuser la sémiotique structurale de beaucoup d'imprécisions et de lacunes dans ses efforts pour théoriser différents domaines de la signification, il nous semble néanmoins qu'elle prouve, justement par des déterminations plus précises qu'ailleurs de la catégorie de l'actantialité, une supériorité assez frappante par rapport à d'autres approches - linguistiques ou conceptuelles. La supériorité de la sémiotique structurale dans ce domaine vient d'une part du fait qu'elle possède un cadre conceptuel de référence plus général permettant de situer un certain type de situations, tel que celui de la mise en norme, par rapport à d'autres types de situations; et d'autre part du fait que ce cadre général possède une organisation, une « grammaire » relativement explicite.

Ceci dit, les situations normatives peuvent être considérées comme partie intégrante de certains processus factitifs qui se composent, à leur tour, de processus simples, de nature intentionnelle. Ces processus simples de nature intentionnelle, qui sont rigoureusement définis par une logique du changement (catégoriel ou graduel) d'état, exigent - pour pouvoir être distingués des processus non-intentionnels («physiques », «spatio-temporels », etc.) - à côté d'un actant d'exécution (appelé aussi «agent »), un actant de contrôle (appelé aussi «destinateur ») : tandis que le premier rôle désigne simplement le fait qu'un objet change, ou change un autre obj et, le deuxième rôle désigne la maîtrise, le savoir, bref : la compétence de changer, ou de changer un autre objet. Souvent ces deux rôles apparaissent en syncrétisme, définissant ainsi le « sujet de faire » en théorie sémiotique, mais rien n'empêche qu'ils soient interprétés par deux instances séparées. II est clair aussi que le contrôle (ou la compétence) d'accomplir une action peut être «effectif » («approprié»); mais il peut aussi être seulement «projeté », «intentionné » sans devoir correspondre pour autant aux exigences propres à tel ou tel changement, à telle ou telle action. Si ce cas se produit, on parlera de processus intentionnels non-contrôlés.

En ce qui concerne maintenant les processus factitifs, ils se distinguent des processus intentionnels simples par le fait qu'ils manifestent deux sujets de faire (le sujet de faire étant considéré, on l'a noté, comme un syncrétisme entre l'actant de contrôle et l'actant d'exécution) dont l'un fait en sorte que l'autre se comporte selon les intentions du premier. Si nous revenons aux deux rôles appelés «autorité de norme » et «sujet de norme», on voit qu'ils sont d'une part des spécifications du rôle «sujet de faire», mais qu'ils ne sont identifiables, d'autre part, que dans un dispositif hié- 
rarchique propre aux processus factitifs (une situation normative ne saurait donc exister dans l'univers des processus intentionnels simples). Les rôles en question reçoivent leur spécification grâce au thème ou propos propre au prédicat dont ils sont des arguments. Le thème du prédicat est paraphrasable comme «transmission d'une norme », ce qui permet d'identifier finalement aussi le troisième rôle actantiel, c'est-à-dire l'objet, qui est précisément la norme.

Comme nous l'avons déjà vu, la «transmission d'une norme » implique un dispositif hiérarchique entre deux sujets de faire, c'est-à-dire l'aptitude d'un sujet de faire à entraîner un autre sujet de faire à se comporter selon un certain plan dont le premier est l'auteur. II s'agit ici d'une condition nécessaire (qui n'est pas d'ailleurs la seule). On peut ainsi prévoir deux types de «déviations » d'une situation normative si ladite condition n'est pas satisfaite : le premier englobe le genre de déviations où un sujet de faire tente de s'ériger en autorité de norme sans en avoir l'aptitude ou la compétence; le deuxième désigne un genre de déviations où un sujet de faire émet une norme d'une manière inintentionnelle.

Une autre condition nécessaire pour qu'une mise en norme réussisse repose sur le fait que le propos véhiculé par une norme doit être adapté à la compétence du sujet de faire qui en est le destinataire. La violation de cette condition entraîne de nouveau un certain type de déviations qui recouvre toutes sortes de nonréponses de la part du sujet de norme en fonction, par exemple, de sa non-compréhension, de son épuisement ou de son écrasement provoqué par la norme dont il est destinataire.

Une troisième condition nécessaire s'applique à la norme et aux modes de sa transmission. II faut que le contenu soit cohérent et non-contradictoire, explicite et exhaustif.

Insistons sur le fait que ces trois conditions sont autant de méta-règles ou «contraintes », et non pas des règles normatives ce qui montre bien l'existence des structures régulatrices au sein même de situations intersubjectives assez complexes.

$\mathrm{Si}$ on considère finalement une situation normative dans le contexte d'autres situations intersubjectives, on peut poser encore une quatrième condition nécessaire pour le bon fonctionnement et la réussite d'une telle situation. II s'agit du fait que la mise en place et l'existence d'une situation normative ne doit pas enfreindre, c'est-à-dire dérégulariser ou destabiliser l'équilibre des situations intersubjectives concernées par la mise en norme du comportement d'un sujet de faire. C'est justement ici qu'un bon nombre de principes moraux, de bienséance, etc. déploient leurs effets. La régularisation de ce genre de situations inter-subjectives extrêmement complexes prévoit en effet le refus de l'application arbitraire des normes, le refus d'un abus de la légitimité ou de la légalité, etc. La violation de cette dernière condition, en compromettant l'inté- 
Peter Stockinger Possibilité d'une représentation conceptuelle de la situation normative
20. A. Weill et F. Terré, Droit Civil. Les Obligations, Paris, Dalloz, 1986. gration de la nouvelle situation normative dans un contexte intersubjectif plus large, amène presque inévitablement ou bien à une dégénérescence de l'équilibre des rapports intersubjectifs touchés (à un «chaos ») ou bien à un nouvel équilibre, qualitativement différent du précédent.

Pour revenir aux trois rôles actantiels «autorité de norme», «sujet de norme » et «norme», remarquons qu'ils peuvent prendre des valeurs différentes selon leur instanciation dans telle ou telle situation normative particulière.

Habituellement, on se représente une situation normative par le fait que les deux rôles «autorité de norme» et «sujet de norme » sont pris en charge par deux acteurs différents :

Acteur $X$ oblige Acteur $Y$ à faire quelque chose.

À côté de l'instanciation des rôles «autorité de norme » et «sujet de norme » en deux acteurs distincts, il faut aussi prévoir des situations d'auto-normativisation, c'est-à-dire des situations où les deux rôles en question s'investissent dans un seul acteur:

Jean s'oblige à prendre la parole.

Un troisième type d'instanciation des deux rôles cités se présente finalement dans les situations normatives réciproques s'exprimant comme suit :

Jean et Paul s'obligent (I'un l'autre) à se rendre service.

II est connu que ce sont justement les situations normatives réciproques qui résultent du contrat au sens classique, c'est-à-dire au sens d'un accord entre deux volontés libres. Les situations d'hétéro-normativisation (instanciation des deux rôles « autorité de norme » et «sujet de norme » dans deux acteurs différents) se manifestent, par contre, dans les contrats «d'adhésion» et les « contrats-type ». Les situations d'auto-normativisation s'expriment finalement dans les contrats avec soi-même, qui constituent un cas-limite en droit 20.

3. Passons à la norme elle-même, au domaine auquel elle s'applique, à sa portée ainsi qu'aux conditions de son application.

Comme nous l'avons vu lors de la discussion de la « visée » de I'acte normatif, le thème d'une norme peut être ou bien un ordre « ouvert » (soit une obligation soit une interdiction) ou bien un ordre « couvert » (une permission).

Maintenant nous allons voir comment d'une part l'obligation et l'interdiction se différencient, et comment d'autre part le statut de la permission devient transparent si on la considère par rapport à un arrière-plan normatif «ouvert», mais partiellement suspendu par l'autorité de norme.

Considérons les exemples suivants :

a) Jean oblige Paul à se rendre à Paris,

b) Jean oblige Paul à ne pas se rendre à Paris,

c) J ean interdit à Paul de se rendre à Paris,

d) J ean interdit à Paul de ne pas se rendre à Paris. 
Ces quatre exemples montrent que (a) et (d) ainsi que (b) et (c) renvoient au même thème, au même contenu propositionnel mais à l'aide de procédures différentes :

- tandis que (a) pose l'obligation directement, (d) l'introduit en tant que résultat d'une interdiction préalable de s'abstenir d'une certaine action ;

- tandis que (c) pose l'interdiction directement, (b) l'introduit de nouveau en tant que résultat - cette fois-ci évidemment d'une obligation préalable de s'abstenir d'une certaine action.

Nous avons donc deux types d'obligations et deux types d'interdictions :

- obligation ouverte (exemple (a)),

- obligation oblique (exemple (d)),

- interdiction ouverte (exemple (c)),

- interdiction oblique (exemple (b)).

Si on prend en considération l'aspect résultatif de ces quatre types normatifs, on peut établir les équivalences suivantes:

$\begin{array}{llr}\text { obligation ouverte } & \simeq & \text { interdiction oblique } \\ \mathrm{O}(\mathrm{p}) & & \mathrm{I}(-\mathrm{p}) \\ \text { obligation oblique } & \simeq & \text { interdiction ouverte } \\ \mathrm{O}(-\mathrm{p}) & \mathrm{I}(\mathrm{p})\end{array}$

Si on passe maintenant au thème de la permission, on voit aisément qu'elle englobe quatre variantes. Ceci est clair si on accepte qu'une permission ne devient saisissable que par rapport à cette toile de fond constituée par l'obligation et l'interdiction - qu'elles soient ouvertes ou obliques. L'identification de tel ou tel type de permission ne peut se faire que grâce à la suspension d'une certaine partie du fond normatif - suspension dont l'auteur n'est évidemment jamais le sujet de norme car dans ce cas-là on n'aurait pas une situation de permission mais une situation de refus. Les quatre variantes de la permission sont les suivantes :

a) Jean n'oblige pas Paul à se rendre à Paris.

(= permission comme résultat de la suspension de $O(p)$ ),

b) Jean n'interdit pas à Paul de ne pas se rendre à Paris

(= permission comme résultat de la suspension de I(-p)),

c) Jean n'interdit pas à Paul de se rendre à Paris

(= permission comme résultat de la suspension I(p)),

d) J ean n'oblige pas Paul à ne pas se rendre à Paris

(= permission comme résultat de la suspension de $\mathrm{O}(-\mathrm{p})$ ).

Étant donné les équivalences que nous avons pu établir entre les différents types centraux du thème d'une situation normative, 
Peter Stockinger Possibilité d'une représentation conceptuelle de la situation normative on peut finalement reconstituer une structure axiomatique de quatre transformations propre à la catégorie de la modalité déontique :

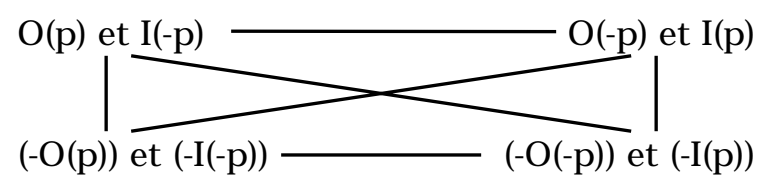

Le domaine auquel une norme s'applique peut être l'action et l'état du sujet de norme ainsi que ses motivations et sa compétence. En plus, elle peut être déterminée par rapport à un lieu et une période temporelle (il faut bien distinguer entre le domaine spatio-temporellement défini d'une norme et l'origine spatiale et/ou temporelle de celle-ci - distinction qui entraîne des conséquences importantes, par exemple en droit civil et commercial).

En ce qui concerne la portée de la norme, elle peut être ou globale ou locale. Dans le second cas, la norme détermine tel ou tel acte particulier, tel ou tel état particulier, etc ; dans le premier cas, elle détermine l'ensemble d'un certain type d'actes, ou d'états.

La portée d'une norme peut finalement encore être définie par rapport à l'axe spatio-temporel, c'est-à-dire qu'une norme peut être valide pendant un certain intervalle partiellement ou totalement déterminé.

Enfin, en ce qui concerne les conditions d'application d'une norme, il y a évidemment lieu de distinguer entre des normes qui s'appliquent d'une manière inconditionnelle et des normes qui n'entrent en existence que du moment où certaines conditions se trouvent préalablement remplies.

\section{V}

Ce sera l'objet d'un autre travail que de considérer les obligations au sens juridique du terme à partir des différentes catégories conceptuelles que nous avons mises en place pour pouvoir représenter les situations normatives au sens «pré-théorique», telles qu'elles apparaissent dans notre langage, nos discours, conversations, actions, etc.

Les hypothèses sous-jacentes à une telle investigation seront, avant tout, les deux suivantes:

a) il y a une certaine continuité entre la compréhension « naïve » de la norme ou de la normativisation d'un comportement, et leurs définitions données en droit ;

b) tandis que la compréhension «naïve » d'une situation normative est très schématique et globalisante, les définitions et procédures juridiques développées pour saisir une telle situation sont analytiques et spécifiques. 
L'hypothèse (a) se vérifierait par la co-présence des différentes catégories conceptuelles dans la compréhension aussi bien «naïve » ou «pré-théorique » que juridique d'une situation normative; I'hypothèse (b) se démontrerait par le fait que les définitions juridiques de tel ou tel type de situations normatives exigent, entre autres, que les catégories conceptuelles dont nous avons parlé soient subdivisées en parties et sous-parties plus précises. 